\title{
Les corporéités de Paris Plages : de la surveillance institutionnelle à l'autodiscipline collective
}

\author{
Benjamin Pradel et Gwendal Simon
}

\section{(2) OpenEdition}

\section{Journals}

Édition électronique

URL : http://journals.openedition.org/tourisme/143

DOI : 10.4000/tourisme.143

ISSN : 2492-7503

Éditeur

Éditions touristiques européennes

Édition imprimée

Date de publication : 1 juin 2014

Pagination : 58-67

ISSN : 2109-5671

\section{Référence électronique}

Benjamin Pradel et Gwendal Simon, « Les corporéités de Paris Plages : de la surveillance

institutionnelle à l'autodiscipline collective », Mondes du Tourisme [En ligne], 9 | 2014, mis en ligne le 30 septembre 2015, consulté le 01 mai 2019. URL : http://journals.openedition.org/tourisme/143 ; DOI :

10.4000/tourisme. 143

\section{cc) (i)

Mondes du tourisme est mis à disposition selon les termes de la licence Creative Commons Attribution - Pas d'Utilisation Commerciale - Pas de Modification 4.0 International. 


\section{Les corporéités de Paris Plages : de la surveillance institutionnelle à l'autodiscipline collective}

\section{Benjamin Pradel}

Sociologue, chargé de recherche, Laboratoire Ville Mobilité Transport

Université Paris-Est Marne-la-Vallée [benjamin.pradel@enpc.fr]

\author{
Gwendal Simon \\ Maître de conférences en aménagement-urbanisme \\ Laboratoire Ville Mobilité Transport \\ Université Paris-Est Marne-la-Vallée \\ [gwendal.simon@enpc.fr]
}

Résumé. Paris-Plages s'affirme comme une organisation socio-spatiale des corps, par des aménagements matériels et symboliques, d'une part, et par un règlement (avec des personnels affectés au maintien de son respect), d'autre part. L'événement est un dispositif prescripteur de pratiques corporelles singulières. II organise la coprésence des corps dénudés selon un principe de stimulation des comportements conformes à une scénographie normative et balnéaire des lieux. Mais cet encadrement institutionnel s'articule avec une autodiscipline collective des corps des "plageurs" dont la coprésence et les interactions contribuent à l'organisation sociale et mimétique du lieu.

Abstract. The Paris-Plages scheme (Paris Beaches) asserts itself as a socio-spatial organisation of bodies through physical and symbolic amenities, as well as through rules of procedure monitored by dedicated staff. The event brings about singular bodily practices. It stages the co-presence of revealed bodies through encouraging behaviours that are consistent with the normative, seaside scenography of the premises. However, this instutional framing interacts with the collective self-discipline of the beachgoers' bodies whose co-presence and interactions are instrumental in the social, mimetic organisation of the venue. 
$\mathbf{L}$ a plage, qui a très longtemps pris la forme d'un désert, ainsi que l'on dénommait auparavant les campagnes que l'on traversait (Desportes, 2005), a été progressivement et doublement investie. La transformation des pratiques sociales liées au loisir a substitué un lieu de plaisir balnéaire au "territoire $d u$ vide" (Corbin, 1988). Le processus continu d'urbanisation a colonisé un monde biophysique en y faisant circuler les composantes de l'urbanité jusqu'à l'artificialisation des plages de bords de mer et leur transposition en ville. Certes, la proximité des liens entre la plage et la ville peut apparaître contre-intuitive tant la plage est l'objet d'imaginaires et d'affects qui la renvoient à une nature "naturelle" par le retour fantasmé à un état de nature (Corbin, 1988). Il reste que le monde urbain s'est imposé à la plage. Il y circule de façon ostensible par les hôtels et les espaces de promenade qui bordent les rivages, et se diffuse en recréant les espaces publics des villes via des régimes de coprésence et de civilités (Coëffé, 2010), à la manière d'un transfert d'urbanité de la ville vers la plage. La réciprocité est aussi vraie, car la plage est devenue "urbaine” en étant mobilisée dans le cadre de logiques festives des villes. Depuis le début des années 2000, des plages “hors sol" sont ainsi créées par et pour les villes, proposant un décor balnéaire simulé. Celui-ci s'exprime fortement par des pratiques sociales et par un rapport au corps - exposé au soleil, dénudé, allongé, vacancier - qui marque les lieux par l'incongruité d'une telle hexis en pleine ville. En effet, rares y sont les lieux qui permettent de bronzer, allongé, immobile, gratuitement, longtemps et en partie dénudé, en dehors des parcs, jardins publics et berges des rivières qu'occupent des citadins héliotropes. À ce titre, les plages urbaines - et Paris Plages en particulier, exemple le plus connu et imité - semblent constituer des tiers lieux innovants, en répondant à une demande sociale par l'organisation d'espaces qui légitiment le dévoilement des corps. Si la plage, en tant que lieu de rencontre idéal entre le corps, la mer, le sable, le vent, le soleil et le vide (Corbin, 1988), raconte l'épopée de la dénudation, totale ou partielle (BartheDeloizy, 2003), les plages urbaines (sans la mer ni le vide) contribuent à cette histoire en l'inscrivant en ville. Les représentations liées à la dénudation des corps activent aisément un registre libératoire (le corps détendu et oisif) lié au relâchement des contraintes du quotidien, qui peut oblitérer les rapports tant sociaux que spatiaux qui enchâssent les corps dans des normes (des corps valorisés ou minorés) et dans des territoires à pratiquer (seul, en couple, en famille...).

À Paris Plages, qui est une plage éphémère organisée par le bureau des événements de la ville de Paris depuis 2001 et qui a attiré quatre millions de visiteurs en 2008, la mise en scène de la balnéarité suscite un comportement collectif normalisé autour, notamment, de l'enjeu d'un dévoilement inédit des corps en ville. Il résulte d'un double "travail”, qui est à la fois celui de l'institution - le traitement de la corporéité dans l'événement par la municipalité - impliquant des dispositifs spatiaux conditionnant les corps, et celui des "plageurs", qui, par le respect de règles implicites, contribuent au fonctionnement du lieu dans une autodiscipline collective des corps. C'est l'hypothèse qui est développée ici : le rapport au corps à Paris Plages s'organise par les règles écrites du lieu imposées par la municipalité (le cadre réglementaire, les comportements pour partie régentés par l'organisation spatiale du lieu et l'instrumentalisation de la symbolique balnéaire) et les règles implicites de la plage, par une coprésence élaborée in situ (gestion des immobilités, des proximités, de l'horizontalité, des nudités). Le corps apparaît alors comme un marqueur du lieu qui, pris dans une mise en scène balnéaire, participe de la construction d'une coprésence aux règles spécifiques. Trois points seront développés : les composants symboliques et matériels servant la reconstitution de l'urbanité, l'encadrement institutionnel des corps puis leur réglage par l'autodiscipline collective.

\section{LA BALNÉARITÉ RECONSTITUÉE}

Les plages urbaines "hors sol" sont le résultat de politiques qui font de plus en plus le choix du "festif" comme levier de développement. En effet, "les villes exercent une attractivité par les opportunités d'animation, permanente ou événementielle, qu'elles concentrent" (Vlès, Berdoulay et Clarimont, 2005). Cette politique 
répond à différents enjeux urbains : donner du sens à une construction territoriale abstraite ou en devenir, médiatiser des projets en cours ou encore dévoiler des aspects délaissés des villes (Pradel, 2008 ; GravariBarbas, 2013). L'univers balnéaire se retrouve instrumentalisé pour recoder, de façon temporaire, un lieu et ses usages. Aussi frappantes soientelles, les plages urbaines ne constituent qu'un continuum de l'artificialisation du balnéaire. Les plages de bord de mer, concomitamment à la massification du tourisme, ont progressivement fait l'objet d'une domestication croissante. Jean Rieucau montre, dans ses analyses sur le littoral méditerranéen depuis l'après-guerre, la manière dont les plages urbaines de bord de mer ont procédé par artificialisation et aseptisation à travers des opérations lourdes de maintenance des plages (réensablement, collecte des déchets, peignage, tamisage et oxygénation du sable). Des aménagements sont réalisés qui voient sable et galets être pulvérisés de produits désinfectants et où "toute discontinuité topographique, morphologique, gênant le cheminement le long du linéaire côtier est gommée" (Rieucau, 2009). Les plages sont surveillées (présence des CRS, de la police municipale, points secours) et réglementées (encadrement des animaux, du feu, du bruit). Elles sont rendues accessibles (rampe pour personnes à mobilité réduite), parkings à proximité), entretenues (nettoyage, plantations, paresable), équipées (douches, cabines, terrains de sport) et "marchandisées" (plages privées, vendeurs ambulants, paillotes). Ces phases d'aménagement et d'embellissement sont révélatrices d'une domestication et d'une mise en scène fonctionnant tel un service pour des populations de citadins (Estebanez, 2006). Cette domestication connaît une sophistication supplémentaire lorsqu'il s'agit d'importer du sable pour élargir un estran (Miami Beach) ou de recréer un rivage dans la perspective d'y implanter des îles artificielles résidentielles (Dubai). La reconstitution artificielle de la balnéarité atteint son acmé avec les parcs à thème, où la recherche mimétique matinée d'embellissement kitsch produit "une sorte d'exotisme à portée de main, pour donner l'illusion du réel" (Brunel, 2007). S'affranchissant des contraintes du site, cette stimulation de la balnéarité crée une ressource de loisir ex nibilo.

Paris Plages reprend la plupart de ces éléments : opération éphémère, elle illustre une mise en scène événementielle dans un contexte de compétition métropolitaine. C'est un outil de réappropriation temporaire de l'espace public et fluvial dans la perspective de renouveler l'attractivité et les images associées à Paris (Fagnoni, 2009). Cette politique du festif doit permettre de "transformer la perception et l'utilisation d'un espace - d'un site muséifié et éloigné à un lieu de représentation à caractère familier, quotidien et approprié" (Pradel, Padeiro et Aguiléra, 2014). Paris Plages aménage la rupture spatiale avec la ville dans la perspective d'une "fantasmagorie de la relocalisation ${ }^{(1)}$ ". Ce n'est néanmoins pas la relocalisation au cœur de la ville d'une plage spécifique, géographiquement localisée, renvoyant à un territoire littoral de référence. Cette politique opère par hybridation : une rangée de palmiers (en pots) évoque la promenade des Anglais à Nice, des cabines rayées blanc et bleu (fermées à clé), des plages du Nord, des oriflammes claquant au vent, les voiles (sans bateau) des ports de plaisance. Des végétaux exotiques convoquent l'imaginaire des plages insulaires tout comme la mise à l'honneur de la Polynésie en 2006 avec faré et fleurs de tiaré. Ici, le sable provient des Yvelines, l'eau douce de la régie Eaux de Paris et les plantes en pots des serres municipales. L'iconographie de l'événement est un mélange qui change chaque année et les activités proposées sont multiples, certaines renvoyant davantage à l'univers de la plage et du littoral (beach-volley, sand-ball, Frisbee, pétanque), d'autres sont plus classiques (taichi, tango/musette, atelier de peinture, concert, bibliothèque). Les pratiques traditionnelles de la plage prennent une saveur particulière : le footing matinal se fait non pas sur le sable mais les pieds sur le bitume; les corps sont rafraîchis non pas dans la mer mais sous les douches et brumisateurs, les jeux de ballon se cantonnent à une zone délimitée sur la place de l'Hôtel-deVille et la brasse à l'air libre se pratique en piscine. Paris Plages fait donc office de simulacre ${ }^{(2)}:$ il organise et encadre ses propres péripéties en 
retravaillant les signes du réel selon une approche kaléidoscopique afin de créer les conditions d'un possible éloignement de l'univers quotidien, tout en étant entièrement ancré dedans et en assumant ce paradoxe apparent. L'opération apparaît alors tel un "îlot d'expérience aux effets de dépaysement" (Ingallina et Park, 2005) à la fois coupé de la ville et entièrement ancré en elle.

Le lieu se constitue par hybridation autour d'une idée de ville associée au quotidien, mais sans le travail, et de l'idée de balnéarité attachée aux vacances, mais sans la mer. Paradoxalement, c'est cette grande absente qui structure les aménagements de Paris Plages. Généralement, les plages sont formées d'une bande de sable pour s'étendre, d'une grève pour marcher et se montrer et de la mer pour se baigner (Urbain, 2002). Elles sont souvent surplombées d'une voie d'accès qui les longe, servant de parking ou de promenade plus ou moins sauvage selon l'environnement, et qui les dessert par des entrées plus ou moins aménagées. Paris Plages s'organise différemment. La Seine est le point d'appui de la métaphore balnéaire, mais la baignade y est interdite. Le parapet et l'étroit trottoir qui la longent sont des lieux d'arrêt momentané pour les promeneurs qui admirent le panorama des façades classées par l'Unesco à défaut d'un lointain horizon. Souvent, les enfants accompagnés s'assoient sur le petit trottoir pour boire à la bouteille, entourés de parents attentifs. Ces arrêts de courte durée renvoient, dans l'imaginaire balnéaire, à cette foule de petites activités les pieds dans l'eau au bord de la mer, souvent le lieu des jeux d'enfants. La route, bitumée, constitue le véritable espace de promenade où se croisent les flux contraires de diverses unités véhiculaires : familles en grappe autour d'une poussette qui ouvre la marche, groupe d'amis en farandole pour fendre la foule, couples se tenant la main, cyclistes et rollers à l'allure saccadée par la fréquentation, etc. Puis vient l'espace de transition, entre promenade et plage, délimité par la rangée de palmiers, lieu de passage entre la station "debout-en mouvement-habillée" à celle "allongéeimmobile-dénudée", là où sont stationnées les poussettes et où les maillots de bain se dévoilent pour rejoindre la plage. Vient ensuite la plage, espace de sable mais aussi de pelouse voire de caillebotis, étroit, surélevé par rapport au bitume, légèrement incliné, limité à ses extrémités par les piles des ponts, ponctué de transats, parasols, fontaines, coincé entre le bitume et le mur de soutènement des quais hauts qui, chauffé par le soleil, renvoie les murmures de la foule et isole le lieu des hauteurs de la ville. Enfin, en surplomb de cet ensemble de lignes redéfinissant la répartition linéaire et traditionnelle des fonctions sur les plages, les quais hauts et les ponts sont des lieux d'observation remplis de badauds et de curieux. C'est ainsi un espace symboliquement clos, qui répartit les corps dans des espaces fonctionnels (promenade, plage, terrains de sports, mobiliers de repos, etc.). L'espace est à la fois réel, constitué d'aména- gements matériels, et idéel car s'y projettent des comportements attendus au service d'une chorégraphie collective travaillant le scénario officiel et, au-delà, l'image de la ville. C'est ainsi que les visiteurs sont considérés comme des acteurs devant animer le décor et faire vivre la dramaturgie du lieu : 'Si la personne n'est pas acteur de ce que j'ai fait, c'est mort. Si elle est spectatrice, ben elle sera sur un Bateau-Mouche ou en haut donc elle voit et après c'est terminé, $y^{\prime}$ a pas d'usage $e^{(3)}$ '. Des règlements et des systèmes de surveillance sont mobilisés pour préserver ce scénario collectif. Participant d'une discipline des corps balnéaires, ils encadrent autant qu'ils produisent la possibilité du dénuement des corps en ville.

\section{LE DÉVOILEMENT ENCADRÉ ET SURVEILLÉ}

Le règlement intérieur de Paris Plages illustre l'injonction paradoxale d'un lieu qui, misant sur la libération des corps via un mimétisme balnéaire et vacancier, encadre leur dévoilement sous couvert d'ordre public. La dénudation est au cœur des attentes de la scénographie et la possibilité de dévoilement, notamment des femmes, devient l'indice de la réussite de l'événement pour son scénographe : "Être en maillot de bain quand tu es une nana seule, faut pas oublier la prouesse! Dans l'espace public quand j'y travaille, la première chose que j'observe c'est la fermme seule. C'est la chose la plus fragile. C'est pour moi l'élément le plus sûr pour savoir si l'espace est 
de qualité, s'il fonctionne, si elle reste, si elle ne se fait pas emm..., si elle est capable de se défaire de ses oripeaux, parce que t'es quand même à poil en pleine ville $e^{(4)}$." L'enjeu est alors de stimuler le mimétisme des corps balnéaires tout en neutralisant les conflictualités potentielles qui peuvent en résulter en ville. Se défendant de jouer au "père la pudeur”, la municipalité a mis en place un premier règlement en 2006 : "Par mesure de sécurité, nous avons décidé d'y interdire les tenues indécentes. Cela aurait pu provoquer des tentations et des comportements dangereux ${ }^{(5)}$. " Le contrôle du rapport corps-vêtement repose sur un argumentaire sécuritaire associant des interdits à des prescriptions aux terminologies imprécises jouant sur des caractères moraux et normatifs : "Le comportement du public doit être conforme aux bonnes moeurs, à la tranquillité, à la sécurité et à l'ordre public [...]. Sont notamment interdits : les tenues indécentes (naturisme, string, monokini, etc. $)^{(6)}$." Face au flou entourant le terme "indécent" et au débat soulevé par celui de "bonnes mœurs", s'est substitué en 2007 un autre texte, proche de celui des parcs et jardins de la ville, stipulant que "le comportement du public doit être conforme à l'ordre public, entendu comme le bon ordre, la tranquillité et la sécurité publics" et imposant "une tenue et un comportement décents conformes l'ordre public”. En signalant qu'une tenue décente doit préserver la tranquillité, l'ordre et la sécurité, la municipalité institue une approche prescriptive et normative. Cependant, il est difficile d'y voir clair dans l'enchevêtrement des injonctions et des tolérances autour de la question du corps dénudé en ville. D’abord, si la nudité à Paris Plages tombe sous le coup de la loi sur l'exhibitionnisme, une semi-nudité peut être potentiellement sanctionnée par une amende forfaitaire de 38 euros, visant par exemple le monokini mais pas le maillot de bain deux pièces. Ensuite, si le règlement de Paris Plages n'interdit pas le maillot de bain, la préfecture de police de Paris rappelle que ce dernier n'est que toléré sur le site et qu'il faut en éviter le port dans les parcs et jardins parisiens. Enfin, le règlement de Paris Plages et la tolérance du maillot de bain ne s'appliquent officiellement que durant l'événement. Pourtant, on constate que les pratiques interdites, comme le port du bikini, ou tolérées, comme le maillot de bain, sont acceptées par les autorités au quotidien sur les quais de Seine car peut-être plus discrètes et moins exposées au public sur des lieux davantage isolés. Et ce qui est interdit à Paris Plages sous couvert d'ordre public ne l'est pas sur les plages du littoral, ce décalage égratignant alors l'imaginaire d'un site qui "donne une image un peu bizarre. [...] sur une plage, il est normal que les gens souhaitent profiter $d u$ soleil et porter un string ou se mettre en monokini $i^{(7)}$ ". La difficulté de comprendre ce qui est interdit, toléré et autorisé en termes de dévoilement et ses limites révèle la volonté de maîtrise municipale des corps balnéaires, car le pouvoir sur ces derniers se situe dans l'interprétation des textes et leur application variable. Ce n'est qu'en 2012 que les premières amendes pour "topless" sont tombées, alors que, avec le port du bikini, observé dès 2007, le topless était pratiqué par des femmes contournant la règle, dégrafant le haut du maillot en restant couchées sur le ventre. Par analogie avec la sociologie des organisations, on peut avancer que ces zones grises autour des limites du dévoilement des corps et des lieux autorisés constituent des "zones d'incertitude" (Crozier et Friedberg, 1977). Bien que flottant, le pouvoir réglementaire de la question vestimentaire permet néanmoins à la municipalité d'asseoir sa maîtrise sur les corps, donc sur les comportements et plus largement sur le déroulement et l'image de l'événement. Les visiteurs sont ainsi pris dans un système de surveillance officielle duquel peut émaner à tout instant au plus la sanction, du moins le rappel à l'ordre, alors même que les règles du jeu ne sont pas claires.

Outre ce cadre réglementaire, il existe un dispositif de surveillance, faisant de l'opération un lieu observé et contrôlé : "Il vaut mieux avoir un arrêt cardiaque sur Paris Plages ou se faire voler son sac à main sur Paris Plages ou perdre son môme sur Paris Plages que n'importe où ailleurs dans Paris (8) $^{(1)}$. Les "yeux" de l'institution urbaine sont légion pour veiller à l'ordre public et à la conformité des comportements et des usages. Régisseurs responsables du fonctionnement des sites, policiers à vélo ou en roller, brigade fluviale, 
secouristes de la Croix-Rouge, agents de la Protection civile, plagistes de la mairie, agents de sécurité, équipes de nettoyage ou membres du bureau des événements de la ville, ils sillonnent Paris Plages tout au long de la journée. Ces agents de l'appropriation tranquille sont identifiés clairement par des tee-shirts aux couleurs de l'opération, badges, uniformes et talkies-walkies. Ils veillent au respect du règlement, incitent, par leur présence, les "plageurs" à montrer qu'ils savent "se tenir" en symbolisant le cadre institutionnel et urbain de la plage. D'un côté, ils facilitent le mimétisme balnéaire en préservant le modèle attendu pour l'image de l'événement, à savoir le corps en maillot de bain, immobile et allongé, offert à la foule. Ils assurent à ce dernier un semblant d'intimité en prévenant les comportements inopportuns, notamment masculins, qui pourraient amenuiser la confiance de la population féminine, qui est au cœur de la "qualité" de l'espace proposé (cf. supra). D'un autre côté, ils exercent un contrôle des corps en imposant les limites de leur dévoilement permis par l'institution, réduisant les possibles par rapport aux plages du littoral. Entre public acteur et comportement déviant, le corps est placé au centre d'une double prise en charge qui participe de l'ambiguité de la plage urbaine. Paris Plages ne relève cependant pas seulement d'un contrôle "par le haut" des corps balnéaires. La surveillance flottante et le flou réglementaire laissent des zones d'incertitude dans lesquelles s'organise

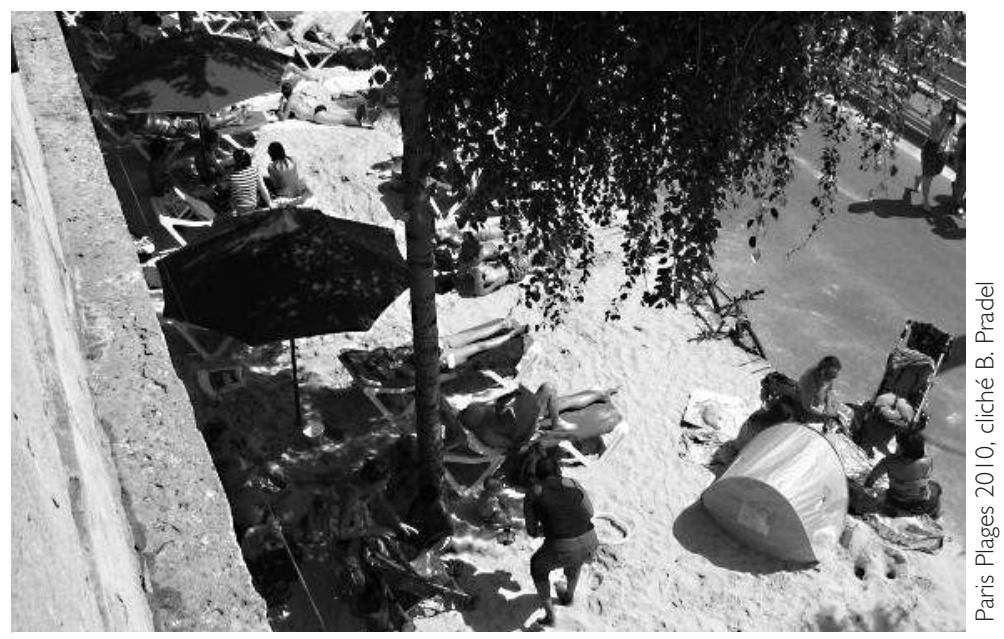

ILLUSTRATION 1• La RÉPARTITION DES POPULATIONS SUR LA PLAGE dÉLIMITE DES ZONES ET DES TERRITOIRES PERSONNELS ORGANISANT UNE IMMOBILITÉ COLLECTIVE PRÉSERVÉE PAR LE RESPECT DE RĖGLES IMPLICITES RÉGISSANT LES DISTANCES, L'HEXIS CORPORELLE, LA LOCALISATION DES ACTIVITÉS

une autodiscipline collective. Celleci repose sur des règles internes au site et aux participants, qui organisent la proximité et la distance des corps balnéaires entre eux. En édictant tacitement les agencements possibles ou incompatibles, ce dispositif régit la possibilité du dévoilement.

\section{LA DISCIPLINE COLLECTIVE DU DÉVOILEMENT}

\section{Zonage et configuration des corps balnéaires}

Les aménagements de Paris Plages divisent l'espace linéaire en autant de séquences d'activités correspondant à des périodes de la journée qui facilitent une discipline collective en distribuant les visiteurs le long du site. Si "la discipline procède d'abord à la répartition des individus dans l'espace" (Foucault, 1975), le principe s'observe à Paris Plages par une disposition des individus qui ne procède pas seulement des aménagements mais également des pratiques collectives. Celles-ci illustrent la manière dont les "plageurs" et les visiteurs gèrent collectivement le dévoilement à travers une organisation spatiale qui peut être analysée à deux échelles. À l'échelle de la plage, les modes d'investissement des lieux par les "plageurs" reposent sur un macro-zonage qui contribue à la préservation de la tranquillité collective, ferment de l'immobilité assumée des corps dévoilés au soleil. Quatre grandes zones se découpent sur les espaces ensablés.

- Les bordures des plages sont des lieux pour des usages temporaires. Elle accueillent des individus qui s'assoient, font une pause dans leur promenade, regardent le panorama, les promeneurs et parfois furtivement les "plageurs", sans trop d'insistance 
toutefois (trop proches des corps alanguis, ils pourraient être pris pour des voyeurs). Ils s'y désaltèrent, discutent et laissent jouer leurs enfants dans le sable avant de repartir ou de s'installer durablement.

- L'avant-scène de la plage, située à distance des bordures, est le lieu où s'exposent préférablement les corps les plus dénudés, pieds vers la Seine, souvent le fait d'un public jeune, entre amis ou en couple. Ici, serviette ou transat sont de mises, les enfants sont peu présents et les tenues vestimentaires légères $(c f$. illustration 1).

- Dans les coulisses, au pied du mur de soutènement ( $c f$. illustration 1), à bonne distance de la promenade, des familles avec nourrissons, groupes d'amis et personnes plus âgées que la moyenne s'adonnent à des activités calmes (pique-nique, tricot, lecture, mots croisés, jeux de cartes, etc.) et, lorsque la plage se rétrécit, ces coulisses n'en sont plus, devenant alors des lieux de bronzage.

- Les enfants se concentrent en bordure, autour des fontaines ou aux extrémités des plages. Les poussettes, nounous et parents signalent ces lieux et en délimitent les contours. Ce zonage permet une surveillance collective des enfants et évite de déranger la quiétude des corps allongés par leurs activités souvent bruyantes et de nombreux déplacements qui font voleter le sable. Ces lieux ne sont pas disputés par les autres "plageurs".

Cette organisation socio-spatiale varie suivant la fréquentation, l'ensoleillement, la largeur des plages, et les zones peuvent parfois s'interpénétrer. Si elle s'appuie sur les aménagements et les caractéristiques du lieu (présence d'un mur, d'ombre, de la Seine, etc.), elle n'en découle pas directement. Elle s'inscrit dans un réglage des proximités et des distances entre des corps vêtus ou dévêtus, en mouvement ou immobiles, âgés, jeunes ou infantiles et par des activités collectives ou singulières. "Les disciplines, en organisant les 'cellules', les 'places' et les 'rangs', fabriquent des espaces complexes" (Foucault, 1975) (cf. illustration 1).

À l'intérieur de ces zones, une autre logique participe d'une discipline collective distribuant les corps. Elle repose sur le partage de règles implicites interprétées comme facilitant le dévoilement des corps et s'observe surtout sur l'avant-scène, où ces derniers sont à la fois les plus dénudés et les plus exposés. Ici, chaque "plageur" se positionne au centre d'un territoire personnel construit en utilisant les équipements fournis (transats et parasols), un ensemble d'objets (sacs, jeux de plage, chaussures, etc.) mobilisés comme des "marqueurs-frontières", avec un marqueur central, généralement une serviette ou un transat. Ces équipements sont agencés selon des distances réglées les uns par rapport aux autres et préservent l'immobilité collective des corps en figeant les positions sur la plage. Ils délimitent une surface qui correspond à celle qu'occupe le corps allongé et sont orientés vers la promenade dans un parallélisme collectif. Immobilité et horizontalité des corps dans ces territoires personnels règlent la chorégraphie collective et garantissent un semblant d'intimité au milieu du rassemblement facilitant le dévoilement. En effet, cette configuration permet une gestion des proximités physiques et une surveillance mutuelle des protagonistes, qui passe par l'importance du regard, à la fois comme élection, menace et vecteur de communication des règles à l'œuvre.

\section{Des corps et des regards}

C'est, au préalable, le regard comme surveillance mutuelle entre les "plageurs" qui est le révélateur d'une autodiscipline des corps dans les espaces de détente. Il spécifie en grande partie le sens social d'un lieu où le "voir et être vu" est central et règle les comportements à travers le croisement des yeux, qui, loin d'être anodin en termes d'interaction, entre élection et menace, "s'enracine dans la possibilité d'un jeu social non verbal de communication" (NahoumGrappe, 1998). D'abord, les "plageurs" cherchent à se protéger des regards du voisinage par cette orientation commune des corps régie par l'agencement des territoires personnels sur l'avant-scène. La configuration limite les points d'observation du voisinage, permet d'identifier les regards latéraux, de réduire les situations de face-à-face, de préserver les parties intimes de la vue et de détecter facilement les regards appuyés. Celui qui ne respecte pas la règle de l'orientation commune est alors collectivement déconsidéré par des regards fuyants, et il est repoussé à une plus 
grande distance physique, son regard pouvant jouer comme une menace potentielle pour l'intimité de chacun (cf. illustration 2). Ensuite, si cette gestion collective de la proxémie préserve l'intimité singulière, elle renforce néanmoins l'exposition aux promeneurs. Il s'agit alors d'exposer son corps entre ouverture et repli (lunettes, baladeur, journal, dos au soleil) pour gérer une immobilité qui, en ville, peut être interprétée comme "un acte délibéré, désiré qui signifie une vacance, une disponibilité, une ouverture au temps et aux mouvements des passants" (Jolé, 2002). Enfin, le regard organise les relations entre les "plageurs" et les promeneurs. Si ces derniers sont dans leurs zones de promenade, à bonne distance des plages, une fausse indifférence mutuelle s'installe car chacun sait qu'il regarde en étant regardé. En revanche, le regard devient un signal des règles internes et implicites aux promeneurs se rapprochant de la plage. Si, par un simple coup d'œil, les nouveaux plageurs-anciens promeneurs jugent mal les distances minimales à respecter, ils risquent les regards désapprobateurs de ceux qui sont déjà installés s'ils venaient à empiéter sur les limites symboliques des territoires personnels. Le regard des "plageurs" allongés dissuade également l'individu qui “entre" sur la plage - à l'arrêt, debout, cherchant un espace, surplombant les corps - de stationner trop longtemps. En effet, par le regard, il s'engage potentiellement dans l'intimité des territoires personnels. Le regard est aussi celui,

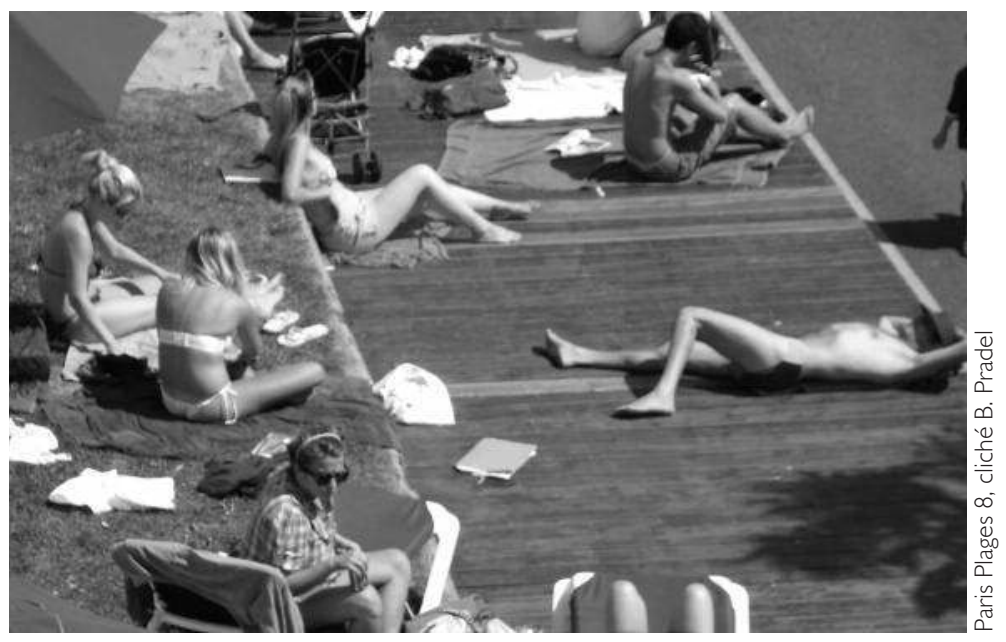

ILLUSTRATION 2 • LA RUPTURE DE LA RĖGLE DE L'ORIENTATION DES CORPS DÉCLENCHE UNE DÉCONSIDÉRATION COLLECTIVE DU DÉVIANT PAR DES REGARDS FUYANTS ET UN ISOLEMENT SOCIAL SUR CETTE PLAGE EN CAILLEBOTIS

collectif, des "plageurs" qui peuvent reprocher tacitement au promeneur, assis sur la bordure ou proche de celle-ci, son insistance éventuelle sur les corps dénudés. Ils l'intiment alors de prendre position dans ou hors la plage en spécifiant son statut par un mimétisme vestimentaire et comportemental. Ici, le regard participe de l'autodiscipline collective par "la mise en corrélation du corps et du geste" (Foucault, 1975).

Le regard orchestre les distances interindividuelles selon des règles implicites partagées concernant l'immobilité et l'horizontalité des corps et la possibilité sociale de leur dévoilement. Cette organisation collective, particulièrement visible sur l'avant-scène des plages, s'articule avec des normes sociales associées au corps et des rapports de genre. Cette organisation révèle d'autres règles tacites sur des attitudes possibles dans ce cadre hybride entre plage et ville. À Paris Plages, des femmes en maillot de bain deux pièces laissent entrevoir une grande partie de leur corps dans un mimétisme balnéaire patent. Ici s'exposent préférablement les corps "beaux" et "libérés", qui doivent être en forme et surveiller leurs formes (Baudrillard, cité par Barthe-Deloizy, 2003). Les personnes les plus dénudées sont celles qui ont le plus préparé leur visite, en adhérant davantage à une scénographie où le corps est surexposé. C'est aussi celles qui cherchent à préserver l'immobilité collective, car le corps trop beau, trop nu, trop exposé provoque chez son propriétaire une moindre liberté de mouvement, le regard des autres glissant moins sur lui (Kaufmann, 200 I). D'autres ne consentent qu'à demi-mot à la scénographie balnéaire, conservant ou aménageant les tenues vestimentaires du quo- 
tidien pour profiter du soleil. Certains ne prévoient que le haut du maillot, conservant un short, un caleçon long, un pantalon retroussé ou une jupe ( $c f$. illustration 2). D'autres, sans maillot, ne relèvent que le bas du tee-shirt jusque sous la poitrine afin de bronzer, voire se mettent en soutiengorge en le cachant néanmoins par un tee-shirt. D'autres enfin, rares, restent entièrement vêtues et en plein soleil, malgré la chaleur. Les rapports de genre soulignent l'inégale occupation de cet espace public balnéaire au cœur de la ville. Les hommes se dévoilent ainsi aisément sur l'ensemble du site (plages, promenades, terrasses, déclivités), qu'ils soient en mouvement ou immobiles, à l'inverse des femmes qui privilégient les espaces de plage et l'immobilité. Elles ne se promènent pas en maillot de bain, évitant de se mélanger aux promeneurs habillés, le bitume marquant un renvoi symbolique à la ville, la norme et l'insistance possible des regards masculins. Certains hommes se fixent ainsi devant les plages, côté Seine, leur regard, allant et venant, étant en partie dissimulé par le flot des promeneurs. Assumer le maillot, pour une femme, nécessite d'avoir sa place sur le sable, dans la collection des corps allongés, assurant sa tranquillité et son anonymat tout relatif. Ainsi, on observe un mélange entre des "plageurs" organisés en maillot de bain, possédant un ensemble d'expédients balnéaires, des "plageurs" à demi habillés, par choix ou par désir de profiter du soleil - par exemple pendant la pause déjeuner pour les travailleurs -, et des "plageurs" habillés qui restent assis au bord ou dans la coulisse des plages. Ensemble, ils colorent l'espace de leurs pratiques entre plage et ville.

\section{Conclusion}

Paris Plages est le reflet d'une culture de l'événement urbain : une réappropriation, par la plage, de l'espace monumentalisé ; un espace public qui produit du rassemblement temporaire sur le mode de la rupture permettant une coprésence de citadins pour partie dénudés ; une sorte d'hybridation de l'espace, entre la plage et la ville, qui repose sur une alchimie entre le registre de la ville "au quotidien" et son revers, une sortie momentanée et ludique qui produit du rassemblement dans un cadre balnéaire recréé. Une plage donc, mais urbaine, scénarisée, technicisée, organisée et surveillée. L'imaginaire et les aménagements proposés illustrent un comportement ambivalent : d'un côté, Paris Plages offre la possibilité officielle de se dévêtir, s'allonger et s'immobiliser dans une forme de libération ludique, balnéaire et temporaire des corps en ville; de l'autre côté, le dispositif scénographique repose sur des usages attendus pour un public acteur tout en étant encadré car considéré comme une foule à canaliser. Les activités et le dévoilement des corps balnéaires sont tour à tour considérés comme une opportunité et un risque dans le scénario événementiel. Opportunité, car au mouvement de la ville s'oppose l'immobilité des corps allongés et en partie dénudés sur le sable, recherchée et importée de la plage par les protagonistes qui rejouent les scènes traditionnelles des vacances balnéaires, essentielles au fonctionnement du lieu. Risque, car Paris Plages est un espace public dans lequel il s'agit de faire tenir ensemble les participants en pacifiant les interactions sociales suivant ce scénario initial de "plageurs" coparticipants. Les logiques individuelles du dévoilement résultent de cette organisation collective institutionnalisée, mais elles émergent aussi des interactions entre les participants, qui s'appuient et instrumentalisent les signaux distillés dans le décor et les choix d'aménagements. C'est dans ce double mouvement que fonctionne l'événement, entre les règles formelles du traitement municipal et celles, tacites, des "plageurs" qui, loin d'être seulement des spectateurs passifs ou extérieurs, sont partie intégrante et active de cette plage "artificielle et surjouée" (Lallement, 2009). Le zonage des plages et l'agencement des territoires personnels, reposant sur le regard comme vecteur de communication des règles et des attentes des "plageurs”, participent d'une autodiscipline collective. Produite par les participants dans les marges et les non-dits du cadre municipal, celleci révèle une balnéarité partagée où chacun s'ajuste aux autres et tient sa place dans une mise en scène collective de la vacance des corps servant celle, instituée, de la ville et de sa municipalité. 


\section{Notes}

( I) "Un lieu qui mobilise l'imaginaire pour mettre en relation avec l'univers entier et sortir des dimensions normales du monde ; en même temps, il se donne comme un lieu convivial, protecteur, quotidien. Les lieux de loisirs sont faits pour attirer, pour donner l'illusion" (Giddens, cité par Bourdin, 2000, p. 93).

(2) Le simulacre s'entend ici comme "une substitution au réel des signes du réel, c'est-àdire d'une opération de dissuasion de tout processus réel par son double opératoire, machine signalétique métastable, programmatique, impeccable, qui offre tous les signes du réel et en court-circuite toutes les péripéties" (Baudrillard, 1985, p. II).

(3) Entretien avec Jean-Christophe Choblet, scénographe de Paris Plages, réalisé le 23 novembre 2006

(4) Entretien avec Jean-Christophe Choblet, scénographe de Paris Plages, réalisé le 23 novembre 2006.

(5) "Les strings interdits à Paris Plages... mais pas les voiles islamiques...", Aujourd'hui en France - Le Parisien, 21 juillet 2006.

(6) Bulletin municipal officiel de la Ville de Paris, $c x \times v^{e}$ année, $n^{\circ} 8$, mardi 25 juillet 2006.

(7) "L'interdiction des strings et des seins-nus à Paris Plages fait débat", LCI-TFI.fr, 30 juillet 2006

(8) Entretien avec Stephane Chave, responsable de la Délégation générale à l'événementiel et au protocole de la ville de Paris, réalisé en novembre 2006.

\section{Références bibliographiques}

Francine Barthe-Deloizy, Géographie de la nudité, Bréal, 2003.

BAUDRILLARD, Simulacres et simulation, Galilée, 1985.

Alain BOURDIN, La Question locale, Puf, 2000.

Sylvie BRUNEL, "Tourisme et mondialisation : vers une disneylandisation universelle?" La Géographie, n 1525, 2007.

Vincent CoËFFÉ, "La plage, fabrique d'une touristi(cité) idéale",

L'Information géographique, vol. 74, n 3, 2010.

Alain CORBIN, Le Territoire du vide.

L'Occident et le désir du rivage, 1750-1840, Aubier, 1988.

Michel Crozier et Erhard Friedberg, L'Acteur et le système, Seuil, 1977.

Marc DESPORTES, Paysages en mouvement, Gallimard, 2005.

Jean ESTEBANEZ, "Les jardins zoologiques et la ville : quelle nature pour le biodôme de Montréal ?", Annales de géographie, n 652, 2006.

Édith FAGNONI, "Plages éphémères et ville durable : l'exemple de Paris", Bulletin de l'Association de géographes français, vol. 3, 2009.

Michel FouCAULT, Surveiller et punir, Gallimard, 1975.

Maria GravarI-BaRBAs, Aménager la ville par la culture et le tourisme,

Le Moniteur, 2013.

Patrizia INGALLINA et Jungyoon PARK, "City marketing et espaces de consommation les nouveaux enjeux de l'attractivité urbaine", Urbanisme, n³ 344, 2005.

Michèle JoLÉ, "Le destin festif du canal Saint-Martin", Pouvoirs, n | | 6, 2002.

Jean-Claude KaUfmanN, Corps de femmes, regards d'hommes. Sociologie des seins nus, coll. "Essais \& Recherches", Nathan, 2001.

Emmanuelle LaLlement, "Paris Plages, une fausse plage pour une vraie ville? Essai sur le détournement balnéaire urbain", Géographie et cultures, n 67, 2009.

Benjamin Pradel, "L'urbanisme temporaire : du court au moyen terme. Une échelle d'action urbaine intermédiaire.", dans. Frédéric de Coninck et José-Frédéric Deroubaix (dir.), Ville éphémère, ville durable. Nouveaux usages, nouveaux pouvoirs, coll. "Formes et figures", L'CEil d'or, 2008.

Benjamin Pradel, Miguel Padeiro et Anne Aguiléra, "Paris sera toujours Paris réflexions sur la centralité dans la métropole francilienne", Métropoles, juin 2014 [http://metropoles.revues.org/4923].

Véronique NAHOUM-GRAPPE, "L'échange des regards", Terrain, n 30, 1998.

Jean RIEUCAU, "Vers des plages urbaines postbalnéaires au début du XXI" siècle. Entre domestication estivale et neutralité hivernale", Géographie et cultures, n 67, 2009.

Jean-Didier URBAIN, Sur la plage, Payot, 2002, 384 p.

Vincent Vlès (dir), Vincent Berdoulay et Sylvie ClarImONT, Espaces publics et mise en scène de la ville touristique, rapport de recherche, Ministère délégué au Tourisme, direction du Tourisme - Laboratoire SET, UMR 5603, CNRS-UPPA, 2005. 